\title{
Measurement of the neutron electric dipole mo- ment by crystal diffraction
}

\author{
V. V. Fedorov ${ }^{\mathrm{a}}$, M. Jentschel $\stackrel{\mathrm{b}}{ }$, I. A. Kuznetsov ${ }^{\mathrm{a}}$, E. G. Lapin ${ }^{\mathrm{a}}$, \\ E. Lelievre-Berna ${ }^{\mathrm{b}}$, V. Nesvizhevsky $\mathrm{b}, \mathrm{A}$. Petoukhov $\mathrm{b}$, \\ S. Yu. Semenikhin $\stackrel{a}{a}$, T. Soldner $\underline{b}$, F. Tasset $[$, \\ V. V. Voronin \\ ${ }^{a}$ Petersburg Nuclear Physics Institute, Gatchina, St. Petersburg, Russia \\ ${ }^{\mathrm{b}}$ Institut Laue-Langevin, Grenoble, France \\ Email: vvv@pnpi.spb.ru (Voronin V.V.)
}

\section{Introduction}

The electric dipole moment of the neutron (nEDM) is a very sensitive probe for $\mathrm{CP}$ violation beyond the Standard Model of particle physics [1,2]. The most precise experiments today use Ramsey's resonance method and ultra-cold neutrons (UCNs) [3,4]. Further progress is presently limited by systematics [5] and the low density of UCNs available. Here we discuss an alternative approach based on spin rotation in non-centrosymmetric crystals.

The statistical sensitivity of any experiment to measure the nEDM is determined by the product $E \tau \sqrt{N}$, where $\tau$ is the duration of the neutron interaction with the electric field $E$ and $N$ the number of the counted neutrons. New projects to measure the nEDM with UCNs aim to increase the UCN density and thus $N$ by orders of magnitude (see [6] for a recent overview). In contrast, experiments with crystals exploit the electric field inside matter, which for some crystals can be by a few orders of magnitude higher than the electric field achievable in vacuum. 
EDM experiments with absorbing crystals were pioneered by Shull and Nathans [7]. Their experiment was based on the interference of the electromagnetic amplitude with the imaginary part of the nuclear one. Abov with his colleagues [8] were the first who paid attention to the presence of a spin dependent term due to the interference of nuclear and spin-orbit parts of the scattering amplitude in the interaction of neutrons with a noncentrosymmetric non-absorptive crystal. Spin-rotation in noncentrosymmetric crystals due to such interference effects as a way to search for a nEDM was first discussed by Forte [9]. The corresponding spin-rotation effect due to spin-orbit interaction was experimentally tested by Forte and Zeyen [10]. The authors of [11,12] have shown that the interference of the nuclear and the electromagnetic parts of the scattering amplitude leads to a constant strong electric field, acting on a neutron during all time of its movement in the non-centrosymmetric crystal. This field was measured in a Laue geometry diffraction experiment [12], in agreement with the calculated value.

The spin rotation can be measured in Bragg [9,10,13] and Laue [14,15,16,17] diffraction geometry. In this paper, we compare both geometries and present preliminary results of a test experiment in Bragg geometry. We show that the sensitivity of an optimized experiment in Bragg geometry can compete with the most sensitive published UCN nEDM measurements.

\section{Comparison of Laue and Bragg diffraction geometry}

A detailed recent study of a nEDM measurement in Laue geometry can be found in [18]. The main advantage of this scheme is the possibility to increase the time $\tau$ of neutron passage through the crystal using Bragg angles $\theta_{\mathrm{B}}$ close to $\pi / 2$ [14]. In this way, times close to the time of neutron absorption $\tau_{\mathrm{a}} \approx 1 \mathrm{~ms}$ were obtained in short crystals [16,19]. However, in order to suppress systematic effects due to the Schwinger interaction (spin-orbit 
coupling), the method relies on an effective depolarization of the neutron beam by Schwinger-rotating the two components of the neutron wave by $\pm \pi / 2$. This fixes the thickness of the crystal to [17]:

$$
L_{0}=\frac{\pi m_{\mathrm{p}} c^{2}}{2 \mu_{\mathrm{n}} e E_{\boldsymbol{g}}} .
$$

$E_{\boldsymbol{g}}$ is the electric field affecting the neutron for the exact Bragg condition for the crystallographic plane $\boldsymbol{g}(\boldsymbol{g}$ is a reciprocal lattice vector) and $m_{\mathrm{p}}$ the proton mass [12]. Therefore the sensitivity of the method cannot be increased by using crystals with a higher electric field $E_{\boldsymbol{g}}$ since the crystal length and thus the time $\tau$ would need to be reduced. On the other hand, it is impossible to increase the sensitivity using Bragg angles extremely close to $\pi / 2$, because of a resulting decrease of the neutron count rate for such angles [18]. In the experiment [18], also a strong sensitivity of the method to crystal deformations was found.

The main advantage of the Bragg diffraction scheme [9,13] is that the electric field acting on the neutron depends on the deviation of the neutron trajectory from the Bragg condition. This allows us to control value and even sign of the electric field and makes new tests of systematic effects possible. On the other hand, the time $\tau$ that the neutron spends in the crystal cannot be increased by using Bragg angles close to $\pi / 2$ as it depends on the total neutron velocity $v$ and not on the velocity component parallel to the crystallographic planes as in the Laue case. However, this disadvantage can be obviated by increasing the crystal thickness, in principle.

A first experiment in Bragg geometry was reported in [10], measuring the neutron spin rotation due to Schwinger interaction in quartz. The experimental value of the spin rotation angle was a few times less than the theoretical expectation [10]. The main experimental difficulty was to obtain monoenergetic neutrons with a well-defined small deviation from the Bragg condition. This was 
solved by placing the monochromator in a strong magnetic field, but this field may cause systematic errors.

Here we propose and use a very simple solution of the problem to obtain these monoenergetic neutrons.

\section{Two-crystal scheme}

Let's consider the symmetric Bragg diffraction case. A neutron falls on the crystal in a direction close to the Bragg one for the crystallographic plane $\boldsymbol{g}$. The deviation from the exact Bragg condition is described by the parameter $\Delta E_{\boldsymbol{g}}=E_{\boldsymbol{k}}-E_{\boldsymbol{k}_{\boldsymbol{g}}}$, where $E_{\boldsymbol{k}}=\hbar^{2} k^{2} / 2 m$ and $E_{\boldsymbol{k}_{\boldsymbol{g}}}=\hbar^{2}|\boldsymbol{k}+\boldsymbol{g}|^{2} / 2 m$ are the energies of a neutron in the states $|\boldsymbol{k}\rangle$ and $|\boldsymbol{k}+\boldsymbol{g}\rangle$, respectively.

In this case the neutron wave function inside the crystal in first order of perturbation theory can be written [20]

$$
\psi(\boldsymbol{r})=e^{-i \boldsymbol{k} \boldsymbol{r}}+a \cdot e^{-i(\boldsymbol{k}+\boldsymbol{g}) \boldsymbol{r}},
$$

where

$$
a=\frac{\left|V_{\boldsymbol{g}}\right|}{E_{\boldsymbol{k}}-E_{\boldsymbol{k}_{g}}}=\frac{\left|V_{\boldsymbol{g}}\right|}{\Delta E_{\boldsymbol{g}}} .
$$

Here $V_{\boldsymbol{g}}$ is the $\boldsymbol{g}$-harmonic of the neutron-crystal interaction potential. For simplicity we consider the case $a \ll 1$, permitting to use perturbation theory.

The electric field acting on the neutron in the crystal is [20]

$$
\boldsymbol{E}=\boldsymbol{E}_{\boldsymbol{g}} \cdot a
$$

where $\boldsymbol{E}_{\boldsymbol{g}}$ is the interplanar electric field for the exact Bragg condition. A nonzero $\mathrm{nEDM} d_{\mathrm{n}}$ results in neutron spin rotation 
by the angle

$$
\varphi_{\mathrm{EDM}}=\frac{2 E \cdot d_{\mathrm{n}} \cdot L}{\hbar v_{\perp}}
$$

where $L$ is the length of the crystal and $v_{\perp}$ the component of the neutron velocity perpendicular to the crystallographic plane. Obviously, sign and value of the electric field (44) are determined by sign and value of the deviation from the exact Bragg condition $\Delta E_{\boldsymbol{g}}$. This is illustrated in Fig. 1.

The presence of the electric field will lead to the appearance of a Schwinger magnetic field

$$
\boldsymbol{H}_{\mathrm{S}}=[\boldsymbol{E} \times \boldsymbol{v}] / c,
$$

where $\boldsymbol{v}$ is the neutron velocity. The corresponding spin rotation angle is [16]

$$
\varphi_{\mathrm{S}}=\frac{2 \mu H_{\mathrm{S}} L}{\hbar v_{\perp}}
$$

Note that the Schwinger effect disappears for Bragg angles of $\pi / 2$ as $\boldsymbol{E} \| \boldsymbol{v}$ in this case:

$$
\varphi_{\mathrm{S}}=\frac{2 E \mu L v_{\|}}{c \hbar v_{\perp}}=\frac{2 E \mu L}{c \hbar} \cot \theta_{\mathrm{B}} \underset{\theta_{\mathrm{B}} \rightarrow \pi / 2}{\longrightarrow} 0
$$

where $v_{\|}$is the component of the neutron velocity parallel to the crystallographic plane. This can be used in the nEDM experiment to suppress systematic effects due to Schwinger interaction.

In the experiment [21] the effect of neutron spin rotation in neutron optics for large $\left(\sim 10^{3}-10^{4}\right.$ Bragg widths $)$ deviations from the exact Bragg condition have been observed. The measured effect has coincided with the theoretical expectation.

In order to tune the electric field $\boldsymbol{E}$, we have to select neutrons with a well-defined deviation $\Delta E_{\boldsymbol{g}}$ from the Bragg condition. For 
this purpose, we use two separate crystals in parallel orientation (see Fig. 2). By heating or cooling the second (small) crystal the interplanar distance $\Delta d$ changes and, therefore, the energy of the reflected neutrons. The second crystal selects only the neutrons corresponding to its own Bragg condition from the whole beam passing through the first crystal. The deviation parameter and, accordingly, value and sign of the electric field having affected these neutrons in the first crystal depend directly on the temperature difference between the two crystals (see Fig. 1).

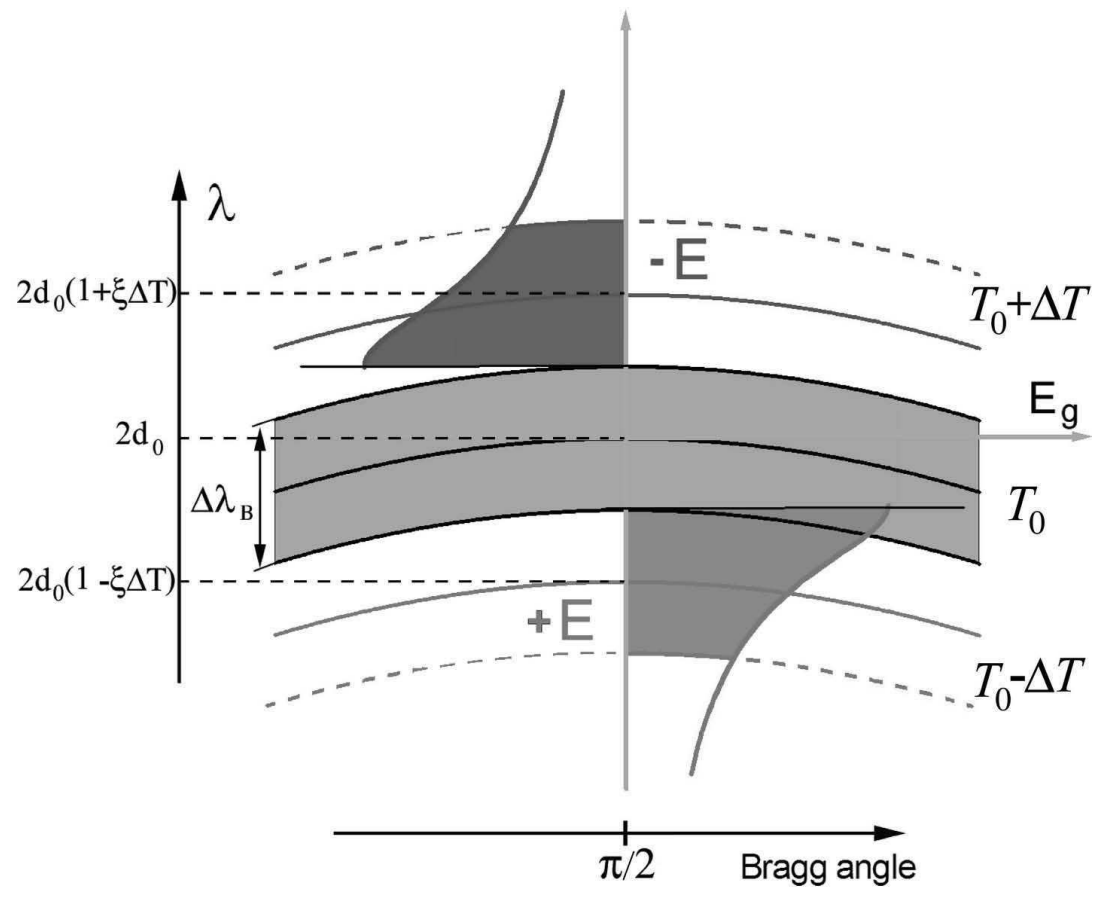

Fig. 1. Electric field for neutrons close to the Bragg condition (Bragg angle $\pi / 2$ and $\left.\lambda=2 d_{0}\right)$ in a non-centrosymmetric crystal: The electric field has a pole for neutrons exactly fulfilling the Bragg condition. By choosing the deviation from the Bragg condition, value and sign of the electric field can be selected. We use Bragg reflection in a second crystal with a different temperature $T_{0} \pm \Delta T$ to select neutrons with a certain deviation.

The value of the Bragg width $\Delta \lambda_{\mathrm{B}}$ for the (110) quartz plane $(d=2.45 \AA)$ is $\Delta \lambda_{\mathrm{B}} / \lambda \approx 10^{-5}$. To shift the reflected wavelength by one Bragg width, $\Delta d / d$ should have the same value, corresponding to a temperature difference of $\Delta T \approx \pm 1 \mathrm{~K}$ (linear coefficient of thermal expansion for quartz $\left.\xi \equiv \Delta L / L \approx 10^{-5} \mathrm{~K}^{-1}\right)$. Note that a common variation of the two crystals' temperatures in itself does not influence the value of the electric field; only 
the temperature difference between the two crystals needs to be controlled.

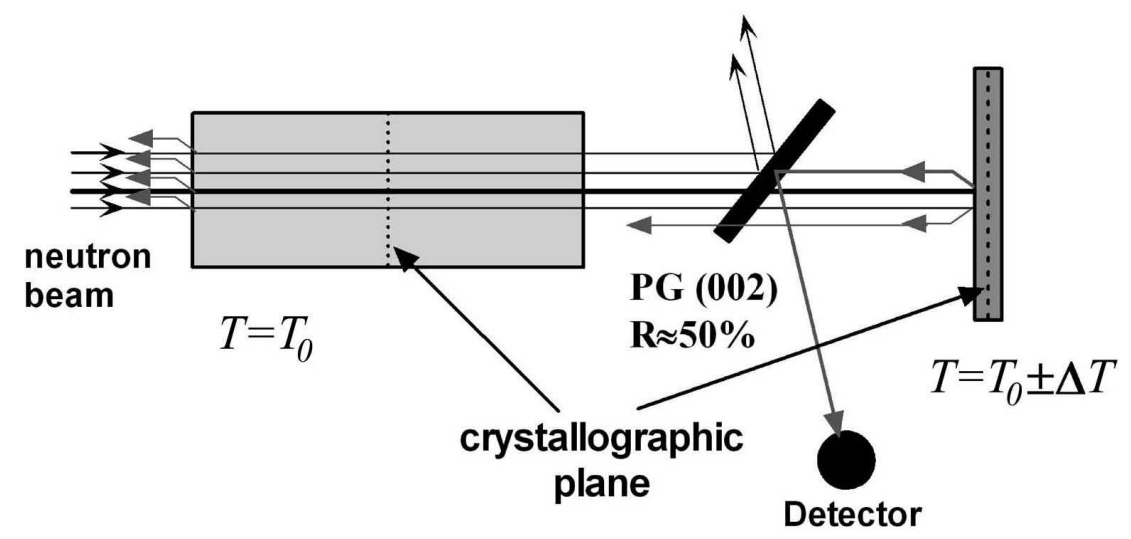

Fig. 2. Two crystals are in parallel position. The crystal deviation parameter $\Delta E_{g}$ for the neutrons reflected by the small crystal is determined by the temperature difference $\Delta T$.

\section{Experimental setup}

A test experiment was carried out at the facility for particle physics with cold neutrons PF1B [22] of the Institut Laue-Langevin. The scheme of the experiment is shown in Fig. 3. We used the (110) reflex of quartz. The length of the main crystal was $14 \mathrm{~cm}$. As mentioned above the backscattering geometry (Bragg angle close to $\pi / 2$ ) allows us to suppress effectively the Schwinger effect.

To measure the nEDM we should direct the initial neutron spin along the $\mathrm{X}$ axis (see Fig. 3) and investigate the dependence of the $\mathrm{Y}$ component of the final polarization vector (i.e. the element $M_{X Y}$ of the polarization tensor) on the temperature difference between the two crystals. The main systematic error of this experiment is due to the residual Schwinger effect. It can be investigated by measuring the polarization component along the $\mathrm{Z}$ axis (elements $M_{X Z}$ and $M_{Y Z}$ of the polarization tensor). Therefore the measurement of a single projection of the final polarization vector only is not sufficient. We used the 3 -D polarization analysis device CRYOPAD [23] to measure the whole polarization 
tensor. In first order the measured difference of the polarization tensor after reversing the electric field is

$$
\Delta \mathbf{M}=g_{\mathrm{n}} \tau_{0}\left(\begin{array}{ccc}
0 & -\left(H^{z} \frac{\Delta \tau}{\tau_{0}}+H_{\mathrm{EDM}}\right) & \left(H^{y} \frac{\Delta \tau}{\tau_{0}}+H_{\mathrm{S}}^{y}\right) \\
\left(H^{z} \frac{\Delta \tau}{\tau_{0}}+H_{\mathrm{EDM}}\right) & 0 & -\left(H^{x} \frac{\Delta \tau}{\tau_{0}}+H_{\mathrm{S}}^{x}\right) \\
-\left(H^{y} \frac{\Delta \tau}{\tau_{0}}+H_{\mathrm{S}}^{y}\right) & \left(H^{x} \frac{\Delta \tau}{\tau_{0}}+H_{\mathrm{S}}^{x}\right) & 0
\end{array}\right),
$$

where $\tau_{0}=\left(\tau_{+}+\tau_{-}\right) / 2, \Delta \tau=\left(\tau_{+}-\tau_{-}\right) / 2$, and $\tau_{+}$and $\tau_{-}$are the times the neutrons stay in the crystal for the positive and the negative electric field, respectively. $H^{i}$ are the components of the residual magnetic field and $H_{\mathrm{S}}^{i}$ the components of the Schwinger magnetic field $\boldsymbol{H}_{\mathrm{S}} \cdot g_{\mathrm{n}}=2 \mu_{\mathrm{n}} / \hbar=1.8 \cdot 10^{4} \mathrm{G}^{-1} \mathrm{~s}^{-1}$ is the neutron gyromagnetic ratio, $H_{\mathrm{EDM}}=E d_{\mathrm{n}} / \mu_{\mathrm{n}}$ the effective magnetic field corresponding to the electric field $E$ and the nEDM $d_{\mathrm{n}}$. For $E=$ $1 \cdot 10^{8} \mathrm{~V} / \mathrm{cm}$ and $d_{\mathrm{n}}=10^{-26} \mathrm{e} \cdot \mathrm{cm}, H_{\mathrm{EDM}}=1.7 \cdot 10^{-7} \mathrm{G}$.

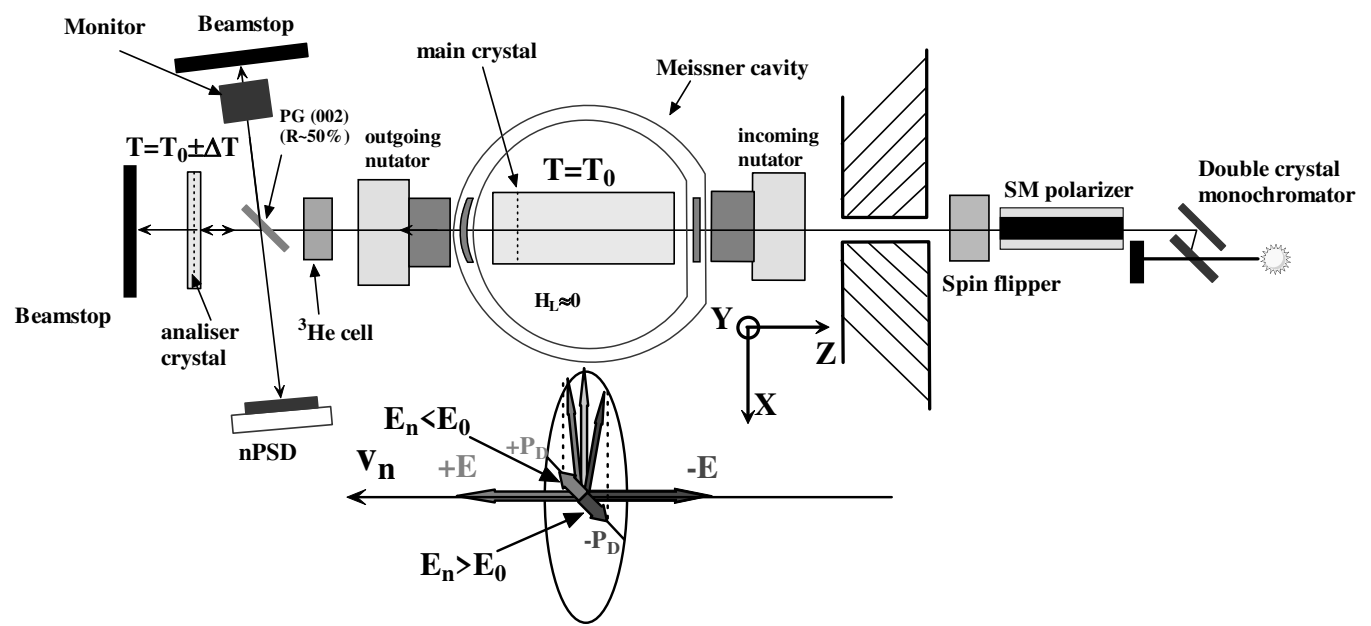

Fig. 3. Scheme of the experiment. The neutrons, coming from the right, pass a pyrolytic graphite monochromator (adjusted to $4.91 \AA$ ), a super mirror polarizer, and a resonance spin flipper. The incoming nutator and a coil inside the Meissner cavity orient the incident neutron polarization vector in space before the main crystal. Correspondingly, a second coil and the outgoing nutator select the component of the outgoing neutron polarization that is analyzed by a ${ }^{3} \mathrm{He}$ spin filter. The analyzer crystal back-reflects neutrons according to its Bragg condition. The pyrolytic graphite PG (002) with about $50 \%$ reflectivity directs the reflected neutrons to the nPSD. It also unavoidably reflects a part of the beam after the spin filter which was used to monitor the beam intensity.

Two main effects can simulate a nEDM: 
(1) Schwinger effect. It results in nonzero components $\Delta M_{X Z}=$ $-\Delta M_{Z X} \approx H_{\mathrm{S}}^{y} g_{\mathrm{n}} \tau_{0}$ and $\Delta M_{Y Z}=-\Delta M_{Z Y} \approx H_{\mathrm{S}}^{x} g_{\mathrm{n}} \tau_{0}$ but does not contribute directly to the components $\Delta M_{X Y}=$ $-\Delta M_{Y X}$ related to the nEDM. However, false effects can arise from imperfections of the $3-\mathrm{D}$ polarization analysis device. These effects can be investigated by measuring the residual Schwinger effect via $\Delta M_{X Z}$ or $\Delta M_{Z X}$. For example, if the angular accuracy of the $3-\mathrm{D}$ analysis is $10^{-3}$ and the Schwinger effect is suppressed down to a level of $10^{-4} \mathrm{G}$ (quite realistic numbers), then the residual false effect will be on the level $10^{-7} \mathrm{G}$, corresponding to $d_{\mathrm{n}} \sim 6 \cdot 10^{-27} \mathrm{e} \cdot \mathrm{cm}$.

(2) Residual magnetic field. Effects due to nonzero magnetic field do not compensate in the difference tensor since the neutrons for both signs of the deviation parameter $\Delta E_{\boldsymbol{g}}$ have slightly different energies and thus spend slightly different times in the residual magnetic field: $\Delta \tau / \tau_{0}=-\Delta \lambda / \lambda$. In our case $\Delta \lambda / \lambda \approx 10^{-5}$. Therefore the residual magnetic field in the polarization analysis device needs to be small:

$$
H^{z} \frac{\Delta \tau}{\tau_{0}} \ll H_{\mathrm{EDM}}
$$

The main idea to control the contribution of the Schwinger effect to the nEDM matrix element $\Delta M_{X Y}$ is to measure the angular, i.e. the spatial distribution of the polarization tensor by a position sensitive neutron detector (nPSD) because the nEDM and Schwinger effects have different angular dependences for Bragg angles close to $\pi / 2$. The $n E D M$ effect is constant, see (15), whereas the Schwinger one is proportional to $\cot \left(\theta_{\mathrm{B}}\right) \approx\left(\pi / 2-\theta_{\mathrm{B}}\right)$, see (8) and would be visible in a spatial dependence of the components $\Delta M_{X Y}$ and $\Delta M_{Y X}$.

\section{Preliminary results}

The measured dependence of the integral intensity of neutrons reflected by the second crystal on the temperature difference $\Delta T$ 


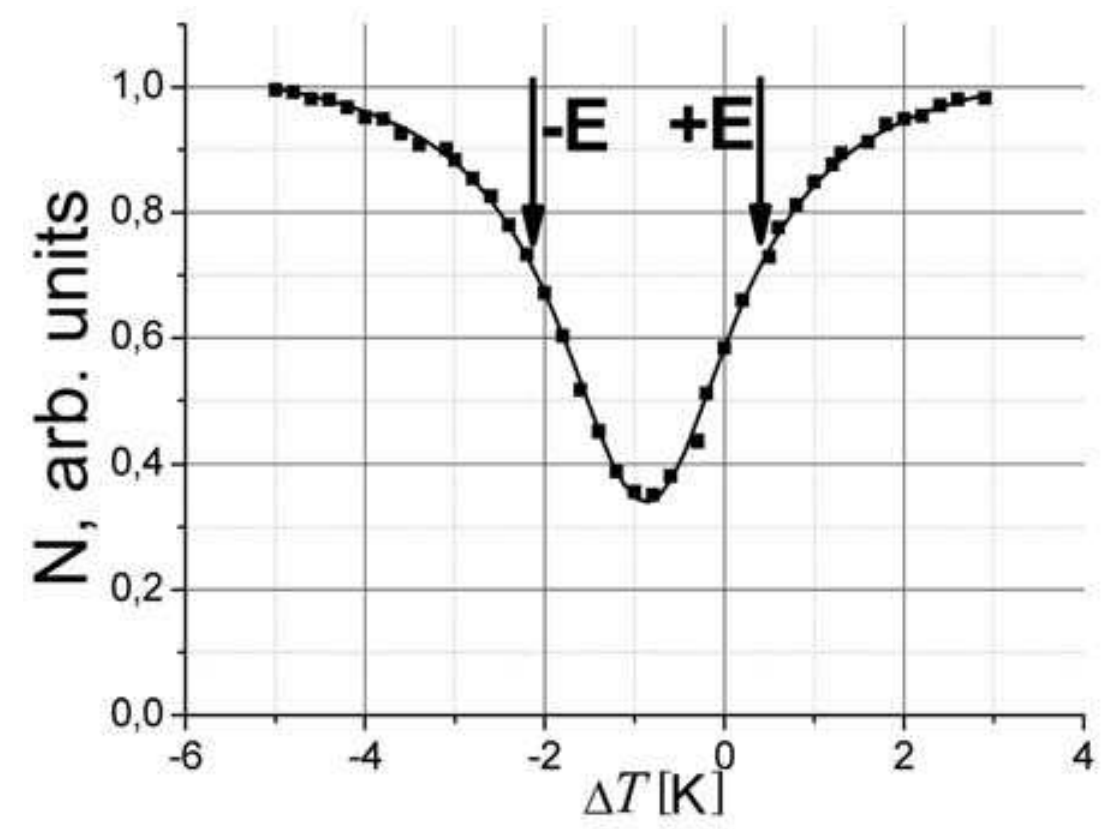

Fig. 4. Dependence of registered intensity of neutrons on the temperature difference $\Delta T$ between the two quartz crystals.

between the two quartz crystals is shown in Fig. 4. The intensity minimum corresponds to equal interplanar distances in the two crystals. The observed difference of interplanar distances for the two crystals at $\Delta T=0$ is probably due to impurities.

In the previous measurements [24] we found the temperature differences corresponding to maximal electric fields acting on the neutron: $\Delta T_{-}=-2.0 \mathrm{~K}$ and $\Delta T_{+}=+0.4 \mathrm{~K}$ (arrows in Fig. 4). The corresponding electric field was determined from the slope of the spatial dependence of $\Delta M_{Z X}$ (see [25] for the graph):

$$
E_{\text {exp }}=(0.7 \pm 0.1) \cdot 10^{8} \mathrm{~V} / \mathrm{cm} .
$$

This value coincides with the preliminary result obtained for the Bragg angle $\theta_{\mathrm{B}}=86^{\circ}$ [24].

The analysis of the spatial dependence of matrix element $\Delta M_{Y X}$ showed no contribution from the Schwinger effect within the present statistics. Summarizing all data, we find the value for 
the angle of neutron spin rotation related to the nEDM:

$$
\Delta \varphi_{\mathrm{EDM}}=(0.9 \pm 2.3) \cdot 10^{-4} .
$$

From this value, we derive the preliminary result

$$
d_{\mathrm{n}}=(2.5 \pm 6.5) \cdot 10^{-24} \mathrm{e} \cdot \mathrm{cm}
$$

The error is only statistical. However, from the preliminary analysis of systematic errors we expect that the result is limited by statistics. The main contributions to the systematic uncertainty are due to the Schwinger effect, the residual magnetic field inside CRYOPAD, a small misalignment between the two crystals, and the curved exit window of CRYOPAD.

\section{Statistical sensitivity of a dedicated setup}

The experiment described above used existing equipment. The count rate in the "gray" position of the polarization measurements was only $\sim 60 \mathrm{n} / \mathrm{s}$ and the statistical sensitivity to the $\mathrm{nEDM}$ was $1.6 \cdot 10^{-23} \mathrm{e} \cdot \mathrm{cm}$ per day. The main limitations were:

- Limited divergence acceptance. The beam divergence accepted by the installation was about $\pm 2.5 \cdot 10^{-3}$ only. However, the beam divergence after the polarizer is about $\pm 1.5 \cdot 10^{-2}$. By installing neutron guides between the different elements, by shortening the installation, and by using a CRYOPAD with larger angular acceptance a factor of $\sqrt{18}$ in statistical sensitivity can be gained.

- Limited beam size. The used beam cross-section $5.5 \mathrm{~cm}^{2}$ was limited by the size of the monochromator, the windows of CRYOPAD, and the size of the crystals used. In a dedicated setup and using larger available crystals, a beam cross-section of $6 \times 12 \mathrm{~cm}^{2}$ is feasible, yielding a factor of $\sqrt{13}$ in statistical sensitivity. 
- Limited crystal length. The interaction time of the neutrons with the electric field can be increased up to the absorption time constant in quartz, by using one large single crystal or a sequence of shorter crystals. We aim for a crystal length of $50 \mathrm{~cm}$ compared to $14 \mathrm{~cm}$ used in the experiment. Taking into account the absorption, this will yield a factor of 2.8 in statistical sensitivity.

- Background, polarization analysis. Optimization of background and polarization analysis can yield a factor of 1.5 in statistical sensitivity.

With these improvements, we expect an improvement of a factor of about 65 in statistical sensitivity, yielding a sensitivity of about $2.5 \cdot 10^{-25} \mathrm{e} \cdot \mathrm{cm}$ per day. This is comparable to state-of-the-art nEDM measurements using UCNs.

\section{Conclusions}

We have measured the nEDM by crystal diffraction in Bragg geometry. Our preliminary result is $d_{\mathrm{n}}=(2.5 \pm 6.5) \cdot 10^{-24} \mathrm{e} \cdot$ $\mathrm{cm}$ (only statistical error). The statistical sensitivity of the test experiment was $1.6 \cdot 10^{-23} \mathrm{e} \cdot \mathrm{cm}$ per day. It can be improved by a factor of 65 in a dedicated setup and can reach $\sim 2.5 \cdot 10^{-25} \mathrm{e} \cdot \mathrm{cm}$ per day for available quartz crystals and neutron beam flux. The present scheme of the experiment allows to control systematic effects related to the Schwinger effect on-line with an accuracy better than the statistical one. The technical requirements (e.g. accuracy of 3-D polarization analysis, alignment of crystals) for a systematic uncertainty on the level of $10^{-26} \mathrm{e} \cdot \mathrm{cm}$ seem achievable [26].

The authors would like to thank the personnel of the ILL reactor (Grenoble, France) for the technical assistance in the experiment. This work was supported by RFBR (grants No 06-02-16378-a, 09- 
02-00446-a).

\section{References}

[1] A. Ritz, these proceedings.

[2] M. J. Ramsey-Musolf, these proceedings.

[3] I. S. Altarev et al., Yad. Fiz. 59 (1996) 1204.

[4] C. A. Baker et al., Phys. Rev. Lett. 97 (2006) 131801.

[5] J. M. Pendlebury et al., Phys. Rev. A 70 (2004) 032102.

[6] R. Golub and P. R. Huffman, J. Res. Natl. Inst. Stand. Technol. 110 (2005) 169.

[7] C. G. Shull and R. Nathans, Phys. Rev. Lett. 19 (1967) 384.

[8] Yu. G. Abov, A. D. Gulko, and P. A. Krupchitsky, Polarized Slow Neutrons, (Atomizdat, Moscow, 1966) 256p. (in Russian).

[9] M. Forte, J. Phys. G 9 (1983) 745.

[10] M. Forte and C. M. E. Zeyen, Nucl. Instr. and Meth. A284 (1989) 147.

[11] V. L. Alexeev et al., JETP 94 (1988) 371.

[12] V. L. Alexeev et al., Nucl. Instr. and Meth. A 284 (1989) 181; JETP 69 (1989) 1083.

[13] V. G. Baryshevskii and S. V. Cherepitsa, Phys. Stat. Sol. b128 (1985) 379.

[14] V. V. Fedorov et al., J. Phys. G. 18 (1992) 1133.

[15] V. V. Fedorov et al., Tech. Phys. Lett. 21 (1995) 884; Physica B234-236 (1997) 8.

[16] V. V. Fedorov et al., Physica B 297 (2001) 293.

[17] V. V. Fedorov and V. V. Voronin, Nucl. Instr. and Meth. B 201 (2003) 230.

[18] V. V. Fedorov et al., Nucl. Instr. and Meth. B 227 (2005) 11.

[19] V. V. Voronin et al., JETP Lett. 71 (2000) 76.

[20] V. V. Fedorov, XXVI Winter LNPI School, vol. 1, (Leningrad, 1991) 65p.

[21] V. V. Voronin et al., JETP Lett. 74 (2001) 251.

[22] H. Abele et al., Nucl. Instr. and Meth. A 562 (2006) 407.

[23] F. Tasset et al., Physica B 267-268 (1999) 69. 
[24] V. V. Fedorov et al., Nucl. Instr. and Meth. B 252 (2006) 131.

[25] V. V. Fedorov et al., in the proceedings of the Panic conference 2008, Nucl. Phys. A, in press.

[26] V. V. Fedorov et al., to be published. 